\title{
High Precision Magnetic Field Measurement for the Muon g-2 Experiment
}

\author{
Peter Winter*广† \\ High Energy Physics Division \\ Argonne National Laboratory E-mail: winterp@anl.gov
}

The Muon g-2 experiment at Fermilab will measure the anomalous magnetic moment of the muon, $a_{\mu}$ with a precision of $140 \mathrm{ppb}$, a four-fold improvement over the former BNL E821 result. If the current discrepancy of about $3.5 \sigma$ between the Standard Model value for $a_{\mu}$ and the former experiments was real, then the new experiment would verify the deviation with $>7 \sigma$ significance. The experiment uses muons with the magic momentum of $3.09 \mathrm{GeV} / \mathrm{c}$ stored in a very homogeneous magnetic field of a $45 \mathrm{~m}$ long storage ring. The determination of $a_{\mu}$ requires the precise knowledge of both the anomalous muon spin precession, $\omega_{a}$, and the magnetic field, $\omega_{p}$, expressed in terms of the free-proton Larmor frequency. The field measurement uses the high precision tool of pulsed Nuclear Magnetic Resonance (pNMR) and requires cross-calibrating a set of probes: a very accurate NMR probe calibrates the 17 probes on the in-vacuum field mapping system (the so-called trolley). This trolley in turn is used to map the field over the full azimuth of the storage ring. The trolley measurement also provides the calibration of the 378 fixed probes around the ring that constantly monitor the field drift. This article presents the summary of the entire field measurement system needed to determine $\omega_{p}$. This work was supported in part by the US DOE, Fermilab and US DOE OHEP under contract No. KA2201020.

ICHEP 2018, International Conference on High Energy Physics

4-11 July 2018

Seoul, South Korea

* Speaker.

${ }^{\dagger}$ for the Muon g-2 Collaboration 


\section{Magnetic Field Measurement for Muon g-2}

The measurement of the muon anomalous magnetic moment is based on the relation $a_{\mu}=$ $\omega_{a} / \omega_{p} \cdot \mu_{p} / \mu_{e} \cdot m_{\mu} / m_{e} \cdot g_{e} / 2$, where the external parameters $\left(\mu_{p} / \mu_{e}, m_{\mu} / m_{e}\right.$, and $\left.g_{e}\right)$ are known to better than $25 \mathrm{ppb}$ from other experiments [1]. The measurement of the anomalous spin precession frequency, $\omega_{a}$, is based on the time and energy spectrum of the decay positrons and is described elsewhere in this proceedings. The measurement of the magnetic field with a systematic uncertainty of better than $70 \mathrm{ppb}$ (due to the very high precision of the pNMR technique the statistical uncertainty is negligible) is achieved through the following key ingredients. Over the course of many months, the field uniformity of the magnetic field was increased through passive and active shimming [2]. This led to an overall dipole field variation of about $\pm 25 \mathrm{ppm}$ as exemplary shown in Figure 1, an improvement of more than a factor of 2 over BNL E821. The average dipole field is stabilized with an active feedback within $\pm 15 \mathrm{ppb}$.

The actual measurement during the physics data taking comprises several ingredients. The drift of the storage field over time is constantly monitored via 378 NMR probes filled with petroleum jelly. Each probe is readout by a waveform digitizer about every $2 \mathrm{~s}$. A tubular in-vacuum trolley system housing 17 NMR probes maps the field at 9000 locations over the full $360^{\circ}$ every $1-3$ days. This calibrates the readback of the fixed probes and provides the azimuthally averaged multipole distributions to better than $30 \mathrm{ppb}$. The NMR readings of the trolley probes are calibrated infrequently against a very accurate standard probe. In a specially shimmed region of the magnet, this cylindrical NMR probe filled with pure water is mounted on a 3-axes automated stage to calibrate each of the 17 trolley probes in-situ. The measured frequency, $\omega_{p}^{\text {meas. }}$, relates to the free proton Larmor frequency via $\omega_{p}^{\text {meas. }}=\left[1-\sigma\left(\mathrm{H}_{2} \mathrm{O}, \mathrm{T}\right)-\left(\varepsilon-\frac{4 \pi}{3}\right) \cdot \chi\left(\mathrm{H}_{2} \mathrm{O}, \mathrm{T}\right)-\delta_{s}\right] \omega_{p}$. The electron screening in water, $\sigma\left(\mathrm{H}_{2} \mathrm{O}, \mathrm{T}\right)$, is known to $2.5 \mathrm{ppb}$ precision. By design of this special probe, its shape factor $\varepsilon$ is well known due to the precision of the cylinder holder. The magnetic footprint of the probe materials, $\delta_{s}$ is minimized by careful design and measured to $1 \mathrm{ppb}$ in a test solenoid at Argonne. The field measurement systems have been successfully commissioned and were performing well during the first physics data taking period 2017/18. Analysis of the data from this Run 1 is ongoing and the systems were upgraded before the Run 2 data taking starting in November 2018.

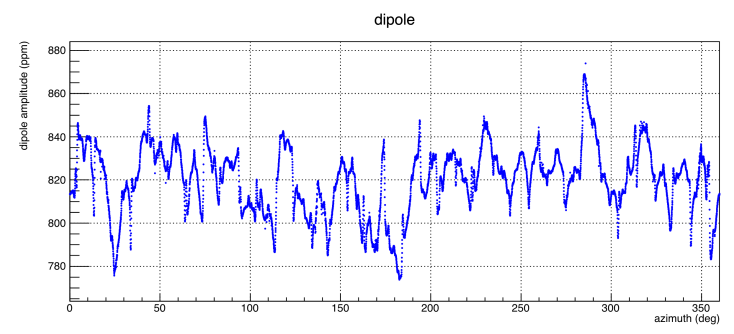

Figure 1: Representative dipole distribution of the storage ring of the Fermilab E989 experiment.

\section{References}

[1] P. J. Mohr, D. B. Newell and B. N. Taylor, Rev. Mod. Phys. 88 (2016) no.3, 035009

[2] J. Grange et al. [Muon g-2 Collaboration], arXiv:1501.06858 